\title{
A Methodology and Framework for Software Memoization of Functions
}

\author{
Pedro Pinto \\ Department of Informatics Engineering, Faculty of \\ Engineering, University of Porto \\ Porto, Portugal \\ p.pinto@fe.up.pt
}

\author{
João M. P. Cardoso \\ Department of Informatics Engineering, Faculty of \\ Engineering, University of Porto \\ Porto, Portugal \\ jmpc@fe.up.pt
}

\begin{abstract}
Enhancing performance is crucial when developing applications for high-performance and embedded computing. It requires sophisticated techniques and in-depth knowledge of the application domain and target architecture. Typically, developers prioritize the application's functional requirements over extra-functional requirements. Thus, a large part of the optimization effort is shifted to performance engineers, who rely on manual effort, alongside many analysis and optimization tools that need integration. This paper focuses on memoization, which caches results of pure computations and retrieves them if a function is called with repeating arguments. We propose a methodology for allowing developers and performance engineers to apply memoization straightforwardly by automating code analysis, code transformations, and memoization-specific profiling. It helps developers with no optimization expertise to quickly set up memoization and, simultaneously, it provides performance engineers with highly customizable analysis and memoization. We provide a concrete implementation supported by a DSL, a sourceto-source compiler, and a memoization framework. We evaluate the methodology and framework with publicly available benchmarks. We show how one can analyze applications to select functions with performance improvement potential, which the experiments reveal might be challenging to find, and improve some applications with minimal effort.
\end{abstract}

\section{CCS CONCEPTS}

- Software and its engineering $\rightarrow$ Software performance; Software development methods; Source code generation; Extrafunctional properties; Development frameworks and environments.

\section{KEYWORDS}

Memoization, Methodology, Source-to-source, Performance

\section{ACM Reference Format:}

Pedro Pinto and João M. P. Cardoso. 2021. A Methodology and Framework for Software Memoization of Functions. In Computing Frontiers Conference (CF '21), May 11-13, 2021, Virtual Conference, Italy. ACM, New York, NY, USA, 9 pages. https://doi.org/10.1145/3457388.3458668

Permission to make digital or hard copies of all or part of this work for personal or classroom use is granted without fee provided that copies are not made or distributed for profit or commercial advantage and that copies bear this notice and the full citation on the first page. Copyrights for components of this work owned by others than ACM must be honored. Abstracting with credit is permitted. To copy otherwise, or republish, to post on servers or to redistribute to lists, requires prior specific permission and/or a fee. Request permissions from permissions@acm.org.

CF '21, May 11-13, 2021, Virtual Conference, Italy

(C) 2021 Association for Computing Machinery.

ACM ISBN 978-1-4503-8404-9/21/05 .. \$15.00

https://doi.org/10.1145/3457388.3458668

\section{INTRODUCTION}

Optimization for performance or energy consumption is a crucial part of the application development cycle, whether the application targets High-Performance Computing (HPC) centers or embedded devices. To achieve performance goals, developers must know an assortment of sophisticated techniques and have in-depth knowledge of the application domain and the target architecture. However, optimization is often not part of the initial development effort for two reasons. First, functional requirements are prioritized over extra-functional requirements, such as performance. Second, developers shift a large part of the optimization effort to performance engineers who know techniques, tools, and target architectures. Along with manual effort, these engineers rely on many tools that sometimes lack integration.

We focus on memoization [6,13], which is complementary to other optimization efforts, such as compiler optimizations, code transformations, and parallelization. With memoization, an application's performance might be improved by caching results of pure computations, and if they are called with repeating inputs, the corresponding result is retrieved from memory instead of performing the computation again.

By applying the typical steps in the memoization optimization process, we propose a methodology that aims to help developers and performance engineers apply memoization for performance improvements. With this methodology, developers can straightforwardly apply memoization or go through optional steps such as analysis and transformation of the source code and memoizationspecific profiling. Therefore, this methodology tackles the problems identified earlier. First, it can support developers with no optimization expertise since it is possible to set up memoization without much effort. Second, it provides an integrated methodology with advanced steps and a high degree of customization for knowledgeable performance engineers.

In this paper, we present a concrete implementation of this methodology using three components. First, we use a source-tosource compiler for the tasks involving the generation and manipulation of the application's source code. Then, to configure the memoization framework and specify analyses and transformations, we use a Domain-Specific Language (DSL). Finally, we use Oddish, a memoization framework that automates the most labor-intensive, time-consuming, and error-prone memoization tasks. Oddish can generate memoization tables very quickly with sound default options, but it also exposes advanced features, such as profiling or approximate computing.

Overall, the main contributions of this paper are the following: 


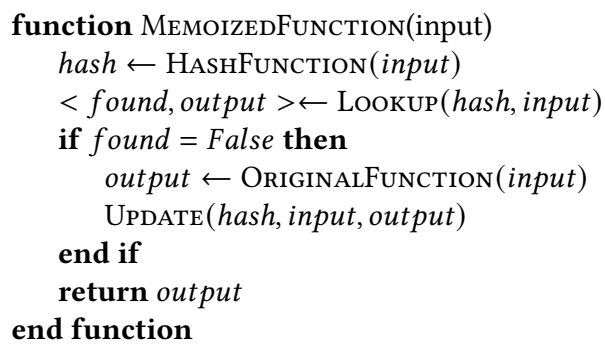

Figure 1: Basic memoization algorithm.

- A methodology with the necessary steps to analyze an application and decide where and how to apply memoization;

- A framework to apply and configure memoization for developers with different levels of expertise;

- A concrete implementation of the proposed methodology and an experimental evaluation of this implementation.

The remainder of this paper is organized as follows. First, Section 2 provides background on memoization. Section 3 presents the motivation for some Oddish features. Then, Section 4 presents our methodology, and Section 5 details the Oddish framework. Next, section 6 and Section 7 describe the experimental evaluation. Section 8 presents relevant state-of-the-art. Finally, Section 9 concludes this paper and presents directions for future research.

\section{MEMOIZATION BACKGROUND}

Memoization $[6,13]$ is an optimization that saves the results of computations for future reuse, by relying on referential transparency [17]. An expression has referential transparency if its value can safely replace it without changing the program's result. The expression needs to be pure, i.e., it must produce the same output for the same input, and it must not have side effects. referential transparency enables optimizations such as parallelization, common subexpression elimination, and memoization.

We can safely cache the results of a pure function. We store the results of its computations and associated inputs, and later on, if the function is called again with repeating inputs, we can return the stored result instead of performing the computation again. Figure 1 presents the basic memoization usage.

Usually, memoization is implemented with a lookup table that stores < input, output $>$ tuples, and is addressed with a hash computed from the function's inputs. Since each target function needs a hash table and logic code to perform lookups and updates, the use of memoization becomes a tradeoff. To achieve speedups, we need more storage space, which may hinder data and instruction caches, and may inhibit the use of memoization in scenarios with limited resources. On the other hand, we also need to consider the execution time overhead. We need to compute a hash, perform a table lookup, and, in the case of a miss, call the original function and possibly update the table. Thus, we need a high hit percentage, or memoization may degrade the original application's performance.

We can also have memoization with prebuilt tables that are not updated, e.g., the results of some trigonometric functions can be tabulated instead of computed. In this case, it may be possible to use perfect hashes if the table is small. We can define several types of memoization based on its configuration. Static, for a prebuilt table without updates, dynamic, for an empty table updated at runtime, and mixed, if it starts prefilled but allows updates with new data.

Memoization has been known for a long time [13] and has been implemented at different levels, ranging from instruction or instruction blocks, in hardware $[1,2,6,7,20]$, to functions, in software $[9,11,12,15,16,18,19,21]$.

\section{MOTIVATION FOR FRAMEWORK}

This section presents the motivation for some implementation choices and features of our methodology and framework.

\subsection{Source-to-Source Compilation}

There are three main reasons to use source-to-source compilation in our proposed methodology. Since we promote customized analysis to enhance the potential of memoization, source-to-source offers two advantages. First, it may be easier for a developer to specify analysis and transformations at the source code level, a more familiar program representation, thus providing a lower entry barrier. Second, some information is lost when the source code is converted into a lower-level representation, which would prevent the ability to express specific analyses and transformations. Finally, sourceto-source compilation allows high flexibility and high integration potential with other tools. After source-to-source compilation, the application can still be compiled with any other compiler or transformed by another approach. This source code portability allows our proposed methodology to fit more use cases and toolchains.

\subsection{Profiling}

This section presents a simple scenario where profiling-based memoization may outperform a traditional approach. Let us consider an application using image processing kernels to process landscape pictures. If we know the type of landscape beforehand and the types and colors of images commonly found, we can set up a memoization table with the most common pixel values. This prefilled table can outperform a traditional approach due to some lookup misses and table updates at runtime.

The first image kernel tested, bw-log, provides dynamic range compression of an image's pixels. It converts pixels from RGB into grayscale and applies a logarithm operator [8], defined as:

$$
O(i, j)=c \times \log (1+I(i, j))
$$

where $c$ is a constant, $I$ is the input, $O$ is the output, and $i$ and $j$ are the image coordinates. The target function in this kernel is log. The second kernel, hsi-single, performs a conversion from RGB into the HSI color space, and its target functions are sqrt and acos.

We compare the original application to five other versions for each kernel. First, two purely online approaches, if-memo [3] and Oddish, which start with empty tables and update them at runtime. Then, three versions of Oddish with profiling support, set up with different image sets: desert landscapes, forest landscapes, and a mix of those. We use the same sets as inputs in a split-validation scheme to ensure that we never test a profiling approach with an image used in its setup. These tests were performed on the desktop computer described as machine $A$ in Table 1. 
version $\square$ if-memo $\square$ Oddish (desert) $\square$ Oddish (empty) $\square$ Oddish (forest) $\square$ Oddish (mix)

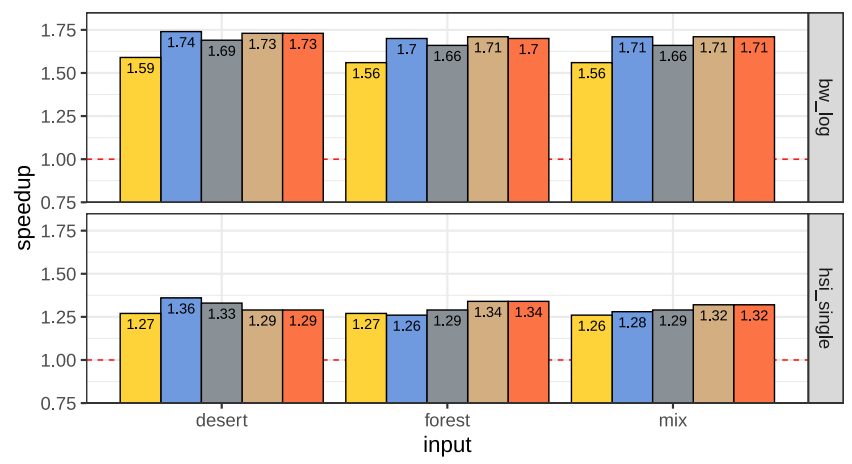

Figure 2: Speedups obtained over the original application by two online memoization approaches and profiling-based memoization approaches using three different training sets for two images kernels, bw-log and hsi-single.

Figure 2 presents the speedups, calculated using the average execution time of 30 runs, obtained by the memoization versions over the original application for the two image kernels. Overall, the profiling-based approach achieves better results across all inputs.

For the bw-log kernel, the profiling speedups are all above $1.70 \times$, while the online speedups are below, $1.57 \times$ for if-memo and $1.67 \times$ for Oddish. Likewise, for hsi-single, we achieve profiling speedups up to $1.36 \times, 1.34 \times$, and $1.32 \times$, depending on the benchmark. On the other hand, the speedups for the online versions never get above $1.27 \times$ for if-memo and $1.33 \times$ for Oddish, and are always worse than the profile-based counterparts. The results for hsi-single illustrate the improvements obtained when the training set matches the input set. For instance, when trained with the desert set and provided with desert images, the profiling-based approach achieved a speedup of $1.36 \times$, against speedups of $1.29 \times$ when trained with other sets, and $1.33 \times$ and $1.21 \times$ for the pure online approaches.

Both memoization approaches obtain speedups for the bw-log kernel, over $1.5 \times$ for if-memo and $1.66 \times$ for the Oddish pure online approaches, and over $1.7 \times$ for all profiling-based approaches. This kernel's target function is log, which is called after the conversion to grayscale. At this stage, the pixels are represented by 8 bits, meaning that all 256 possible pixel values will fit in both approaches' memoization tables (65536 capacity, in this case). This fact explains why the profiling-based approaches, regardless of the training set, achieve the same average speedup over the original, $1.72 \times$. In this kernel, the main difference between the two approaches is that the online approach starts with an empty table, leading to some initial misses. The consequences of a miss are two-fold, and they may explain the difference in performance. First, we need to call the original function, log and then we need to update the table with the result we just computed.

The online approach can be more flexible since it is possible to train a profiling-based approach with non-representative data. For instance, in hsi-single, when the profiling-based approach is setup with desert images and provided with forest images, it has worse performance than both pure online approaches. Despite a small difference, the online approaches update the tables at runtime and adapt to different scenarios.

\section{A MEMOIZATION METHODOLOGY}

We present a methodology to enhance an application's performance through the use of memoization. By following these steps, developers can easily apply this technique to their applications, improving their performance with possibly low effort. This methodology's high degree of automation allows developers with different expertise levels to benefit from memoization. However, more experienced developers, or those more knowledgeable about the application and its inputs, will achieve maximum benefit from memoization due to the optional steps of analysis, profiling, and overall tuning.

Our proposed methodology decouples the original application's source code from the code needed to apply memoization. Program analyses and transformations are specified in files separated from the application code and then joined via a source-to-source compiler process similar to weaving from Aspect-Oriented Programming (AOP) [10]. This separation of concerns promotes better maintainability, extensibility, and reusability. On the other hand, this also allows for co-design and co-development of applications. The domain expert can write the application, and the performance engineer or target hardware expert can write optimization code that takes advantage of the characteristics of the target architecture.

With this methodology, we intend to ease the application of memoization as much as possible, especially by automating timeconsuming and error-prone processes. Due to the use of a source-tosource compiler, we can automate all memoization code and report generation, as well as developer-defined analyses and transformations on the original application.

We use three specific components to support a concrete implementation of this methodology. First, we needed a source-to-source compiler to interact with the application's source code, and we decide to use Clava [4]. Second, we needed a mechanism to give developers the control of the source-to-source compiler by defining their strategies, and we decided to use the LARA DSL [5, 14]. Finally, we needed an automated and customizable memoization framework, and we developed Oddish, which Section 5 details.

\subsection{Methodology Flow}

Figure 3 illustrates our methodology's flow. We start with the original application and may follow multiple paths to the memoization code generation (3). We can analyze the application first (1) to reveal, e.g., the application's hotspots and focus optimization efforts on those functions. In the optional profiling step (2), we instrument the application to collect memoization data (a) from target functions. The following paragraphs detail these activities.

4.1.1 Analysis. In this optional step, we gather static and dynamic information about the application to guide, control, and improve how memoization is applied. For instance, at this point, we find and filter memoization candidates, possibly with the memoization framework's help. Whenever a developer is trying to optimize an application, it is crucial to find the application's hotspots to focus the bulk of the optimization effort where it may have more impact. We can discover these hotspots through analysis, either by instrumenting the code to measure execution time or by loading 


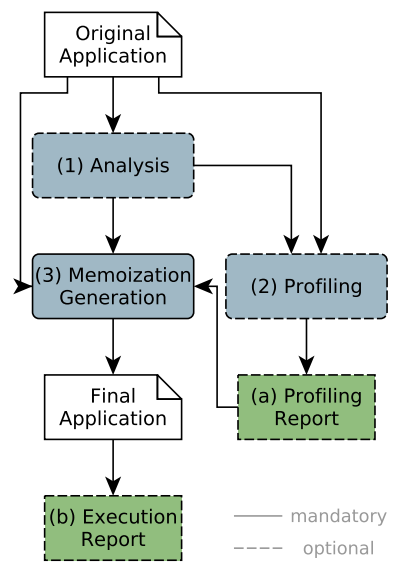

Figure 3: Main flow of the memoization methodology.

reports from external profilers. We can also ignore this step and instruct the memoization approach to target every memoizable function. It may also be possible to find regions of code that exhibit referential transparency [17] using dataflow analysis. After finding these regions, it may be possible to extract them through function outlining and apply memoization to the newly generated function. Another possibility lies in static or dynamic range analysis to provide valuable insight into the range of values an input may take and, by extension, on the size of the memoization data structures.

4.1.2 Profiling. While the Analysis step addresses other profiling efforts, we perform profiling tailored to collect memoization data in this optional step. One of this methodology's key features is that we may use profiling to find which values should prefill the memoization data structures, possibly avoiding issues such as frequent eviction of highly-repeating inputs whose hashes collide. With profiling, it is possible to know which of these inputs are expected to repeat more and fix them on the memoization table. Another issue tackled by profiling is that we may avoid unnecessary updates to the memoization data structures. At the start of the execution, we already have a prefilled memoization structure, avoiding initial misses and updates. Our profiling data allows us to estimate the hit rate of the final generated table and, if it is above a satisfactory threshold, we can disable the update logic, leading to better-performing applications. There is a noticeable overhead for profiling. It involves several executions of the target application with representative inputs and analysis of the corresponding profiling reports. However, this is common to any profiling approach, and we consider it a one-time cost operation, which may provide performance improvements once we deploy the final application.

4.1.3 Memoization Generation. This final step generates the memoization code and produces the final application to deploy in a production scenario. Here we can control several parameters such as which functions to target and how memoization is applied. We can also provide memoization profiling reports at this step, which are taken into account to prefill the memoization data structures. At the end of the optimized application's execution, we may optionally generate an execution report for manual inspection.

\section{ODDISH MEMOIZATION FRAMEWORK}

Oddish provides developers with a wide range of customizable features to apply memoization, from profiling to approximation and the possibility of finding pure function targets and dealing with multiple-output functions.

It is very straightforward to apply a simple memoization strategy with Oddish. First, we define the memoization targets or let Oddish find them. Then, Oddish will generate a wrapper, an empty table, and all needed logic to update it at runtime. Oddish has two options to control whether it updates the table at runtime. In case of a miss, if the table is online, Oddish will try to update it with the result returned by the original function. If the table position is empty, we store the result. If it is not, an update may still occur if the alwaysupdate option is turned on. The default Oddish configuration is an empty table, online, and with update-always turned off, which avoids replacing older values in the case of a hash collision.

We implemented Oddish as a LARA library for Clava that a developer can import into any LARA code. Oddish also includes a $C$ library that should be linked to an instrumented application when we use memoization profiling. This library collects runtime information about function calls and their inputs and generates profiling reports. In the remainder of this section, we detail some of the Oddish framework's main features and implementation choices.

\subsection{Implementation}

Our implementation of memoization in Oddish uses hash tables to store function computation results and associated arguments. The size of the hash tables is provided by the developer and is given by $N=2^{B}, B \in[1 \ldots 16]$. Our tables are bi-dimensional $C$ arrays with $N$ rows and $C$ columns. If $I$ and $O$ are the numbers of inputs and outputs of the function, then $C$ is given by $C=I+O$. Since each element is stored as 8 bytes, the total amount of bytes needed for a memoization table is given by $S=N \times C \times 8$. For instance, a table with 65536 elements for a function with a single input and a single output requires $1 \mathrm{MB}$ of memory.

Oddish uses a simple hash function $[3,19]$ to address the memoization table. First, it performs XOR operations on all the function's inputs, with the result having the same size as the largest type received by the function. Then, it splits this result in half into its Most Significant Bits (MSB) and Least Significant Bits (LSB) components, and it performs another XOR on these. The hash function repeats this operation until the result has size $B$, the number of bits needed to address the table, masking some LSBs if $B$ is not a power of 2 .

To keep our code as clean as possible and allow further manual inspection or even change, Oddish performs the memoization operations in target-specific wrappers, such as the one presented in Figure 4. It replaces calls to target functions with calls to newly generated wrapper functions. These wrappers contain all the memoization logic, including the hash computation, table lookup, and, in the case of a miss, a call to the original function and table update.

Currently, Oddish is limited to int, float, and double data types, although it should be straightforward to extend support to other types. Another current limitation is the number of parameters supported. When using Oddish to find memoization candidates, it considers only functions with a maximum of 3 parameters, as empirical testing reveals that as the number of parameters grows, 


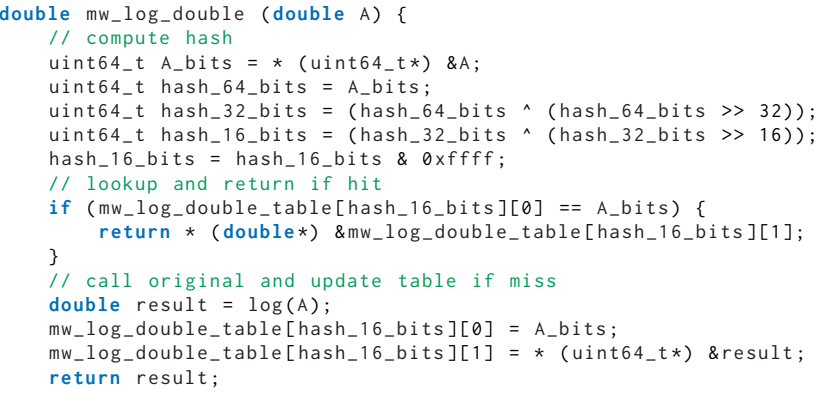

Figure 4: A wrapper function for log generated by Oddish with all the memoization logic code.

the probability of getting a hit on the table diminishes. However, this constrains only the automated target search, and a developerdefined target can have any number of parameters.

\subsection{Profiling}

Traditionally, memoization approaches build their lookup tables at runtime, but Oddish also supports profiling-based memoization, one of the main features of this framework. Oddish can automatically instrument the application to use a C library (also part of Oddish) to collect runtime data about function calls and their inputs, guiding the decision of which values to use. Since it is crucial to profile an application with representative data, several runs with different inputs may be necessary. Each of these runs generates a report, which Oddish can automatically merge.

Although Oddish generates all the code for the developer, it is still possible to control several parameters that affect what is profiled and how it is analyzed. In addition to specifying which functions to profile, it is possible to choose whether we profile them globally or locally, i.e., if we want to analyze all calls to a target function together or make a distinction based on the call site. Different call sites may have different contexts and repetition profiles, which we use to decide precisely where to apply memoization.

Oddish enables the definition of heuristics at different granularity levels to control how the report is analyzed and how it generates the memoization table. It is possible to skip an entire target function or a specific call site, e.g., if there were only a minimal number of calls or low input repetition. It is also possible to skip individual < input, output $>$ tuples of the profiling report. Finally, we can specify how to prioritize entries in a collision, e.g., prefer the entry with higher average repetition across all profiles or with less variance in repetition.

\subsection{Other Features}

Oddish has utility methods to find memoization candidates using a set of heuristics. If requested, Oddish can generate a report with information about functions that were considered but not chosen as targets, such as the heuristics that failed. Through LARA, developers can develop heuristics and filter the found targets, e.g., using previous profiling data to focus on hotspots.
Oddish allows memoization for functions with multiple outputs (through pointers). Although these functions are not found automatically by Oddish, developers can profile and generate memoization tables for them. These functions must follow the convention that all inputs appear before the outputs in the parameter list.

The Oddish framework can use approximate memoization and treat similar inputs as equal, increasing the number of hits at the expense of accuracy. This tradeoff is entirely dependent on the goals and can rely, e.g., on a Pareto Frontier. Thus, Oddish does not perform any accuracy measurements or tuning but leaves this at the developer's discretion. The implementation masks some bits of the mantissa, according to the IEEE-754 Floating Point standard binary representation.

\section{SYNTHETIC BENCHMARK EVALUATION}

This experiment uses a controlled environment to show the effects of specific input characteristics on performance and how Oddish can be used to analyze an application and capitalize on performance improvement opportunities.

\subsection{Experimental Setup}

We calculated the presented speedups using the average execution time of 3000 runs, performed on a desktop computer with specifications described in Table 1 as machine $A$. We configured Oddish to use profiling data from previous runs and start with a prefilled, 65536-element table that is not updated at runtime. The two Oddish parameters tested are whether to consider call sites globally or individually for the same target function and an application policy, a predicate that Oddish tests on the profiling data. In this case, the predicate is a threshold for the generated table's expected hit rate.

The tested benchmark replicates an image processing application. Initially, the kernel converts the image's RGB components into grayscale and then applies a logarithm operator, defined in Equation (1). There are two different call sites in this experiment, each calling the target kernel with different inputs.

In this experiment, we control three characteristics of the inputs. The input size, $T$, is relevant as larger inputs will not entirely fit in the table. The percentage of repetition, $R$, indicates how many calls took repeating inputs. If there are many more unique values than the table can hold, the final hit rate is limited by the table's size rather than the input's repetition rate. Finally, the repetition's distribution, $D$, is how spread out the repetition is along the set of unique input elements. We automatically generated inputs with combinations of these characteristics, consisting of evenly distributed points between randomly chosen lower and upper bounds. The size of both inputs is $T=1000000$, and the repetition distribution is $D=$ $10 \%$. The input data repetitions, $R 1$ and $R 2$, assume the extreme values of $0 \%$ or $100 \%$. These inputs illustrate uncommon scenarios in traditional benchmark suites.

\subsection{Experimental Results}

Figure 5 presents the speedups obtained with profiling data. The apply policy is represented in the X-axis, always will always apply memoization, and over $50 \%$ will only apply memoization if the expected hit rate is above that percentage. The expected hit rate is calculated based on the inputs inserted in the generated table from 


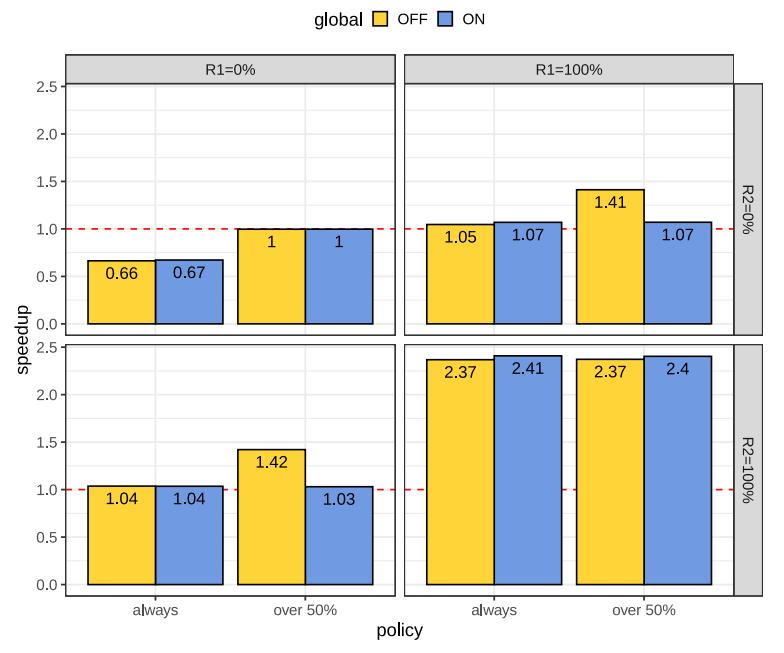

Figure 5: Speedups obtained with profiling data, for different policies and per-site decisions, over the original version.

those observed during profiling. The chart series represents whether Oddish performs this analysis and subsequent code generation considering targets globally, global on, or individually, global off.

The effects of the application policy can be seen when neither of the profiled inputs repeats, $R 1=0 \%$ and $R 2=0 \%$. Oddish can insert some of the input values in the generated table, but given the large size of the input compared to the table, the expected hit rate is only $6.55 \%$, which is not enough to achieve speedups. If we always apply memoization, we get a slowdown of around $0.66 \times$, while if we only apply it if the expected hit rate is above $50 \%$, the performance unaltered, as memoization is not applied. These results show that if profiling data predicts slowdowns for a specific target function, we can skip it and still use memoization elsewhere.

Oddish can also control which individual call sites to target for the same function. We see this configuration's effects when one of the profiled inputs exhibits $0 \%$ repetition and the other $100 \%$. In these cases, if we look at both sites globally, the expected hit rate is $53.28 \%$. If we consider both sites individually, in the site where the profiled input repeats $0 \%$, the expected hit rate is $6.55 \%$, and in the site where the profiled input repeats $100 \%$, the expected hit rate is $100 \%$. There is no difference between treating call sites globally or individually if the application policy is always to apply memoization, and we reach speedups of $1.04 \times$. However, when defining a hit rate threshold, per-site profiling becomes essential. Given the expected hit rates, it is more profitable to target only the high-repetition site rather than both sites, as we can see by the achieved speedups, $1.42 \times$ compared to $1.03 \times$.

These results show that inputs can have behaviors that are difficult to predict, which hinders the benefits from memoization. Our methodology helps developers test these corner cases, reveal these behaviors and choose the best outcome.

\section{EXPERIMENTAL EVALUATION}

In this section, we apply simple memoization strategies to a set of benchmarks to show that it is possible to improve an application's performance with minimal effort by letting Oddish discover memoization targets and generate all code.

\subsection{Experimental Setup}

With the default configuration, Oddish starts with an empty memoization table, which is updated at runtime. We test several variations based on the chosen targets functions. With all, we apply memoization to every target function found, and with math, we target only functions from the $\mathrm{C}$ math library. Finally, with selected, we target selected functions for each benchmark, simulating the scenario where the developer knows which functions have potential for memoization. In this case, it translates to functions with input repetition over $10 \%$. In these tests, we use two table update policies. The default option, UA-OFF, only updates the table if the corresponding position is empty, while UA-ON always updates the table, possibly evicting a previously stored result.

The presented results show the speedups obtained over the original versions of the benchmarks, computed from the average execution time of 10 program runs. For comparison, we also present the results from if-memo [3], a recent memoization approach that also targets functions to provide software-level memoization, although with less depth of configuration and without profiling capabilities.

Table 1 presents the main characteristics of the two desktop computers used in these experiments, including relevant hardware, software, and compilation framework. The benchmarks were compiled with the flags -03 -march=native - flto.

We performed our experimental evaluation with 11 benchmarks from different suites. To contextualize the speedups obtained with Oddish, we calculated the Lower Bound (LB) and Upper Bound (UB)

Table 1: Specifications of the machines used for testing.

\begin{tabular}{lll}
\hline & machine A & machine B \\
\hline CPU & Intel Core i7-9700 CPU & 2x Intel Xeon E5-2630 v3 \\
CPU Frequency & 3.00GHz & $2.40 \mathrm{GHz}$ \\
RAM & 16GB DDR4 & 128GB \\
OS & Ubuntu 20.04 LTS & Ubuntu 16.04 LTS \\
Compiler & GCC 9.3 & GCC 7.3 \\
libm & v2.31 & v2.23 \\
libomp & v4.5 & v4.5 \\
\hline
\end{tabular}

Table 2: Lower and Upper Bounds for each benchmark.

\begin{tabular}{lrrrrr}
\hline \multirow{2}{*}{ Benchmark } & \multicolumn{2}{c}{ machine A } & & \multicolumn{2}{c}{ machine B } \\
\cline { 2 - 3 } \cline { 6 - 6 } & LB & UB & & LB & UB \\
\hline ATMI & 0.81 & n/a & & 0.94 & 5.64 \\
barsky & 0.90 & 1.20 & & 0.84 & 1.61 \\
basicmath & 0.98 & 1.00 & & 0.98 & 1.01 \\
equake & 1.00 & 1.05 & & 0.99 & 1.14 \\
FFT & 0.96 & 1.17 & & 0.94 & 2.02 \\
fmm & 1.00 & 1.02 & & 0.92 & 1.05 \\
ocean_cp & 0.99 & 1.05 & & 0.99 & 1.18 \\
sar & 0.91 & 1.74 & & 1.00 & $\mathrm{n} / \mathrm{a}$ \\
susan & 1.00 & 1.00 & & 1.00 & 1.00 \\
typeset & 1.00 & 1.00 & & 0.99 & 1.00 \\
water_spatial & 0.95 & 1.11 & & 0.99 & 1.24 \\
\hline
\end{tabular}


for each application and present them in Table 2. We calculate LB by running a version of the application where every call target functions results in a miss, defaulting to the original function with the additional Oddish overhead. We could not adopt a similar approach to the UB calculation and force a hit on every call since we would have to return dummy data, which alters some benchmarks' behavior. Therefore, we assume the unrealistic scenario where the target functions are so optimized that their computation becomes instant. We calculate UB by removing the time of the targeted functions, $t_{t}$, from the total execution time, $t_{o}: U B=\frac{t_{o}}{t_{o}-t_{t}}$

\subsection{Experimental Results}

Figure 6 presents the speedups obtained with the memoization versions over the original versions of each benchmark. Overall, we can achieve modest speedups in some of the benchmarks. It becomes clear that no single configuration is the best for all benchmarks and that some benchmarks have minimal potential, as seen in Table 2 .

We achieve the best results in ATMI and FFT overall, equake for machine $A$, and water_spatial for machine $B$. ATMI presents the best results, with speedups up to $1.13 \times$ and $1.55 \times$, depending on the machine. We have better results with $U A-O F F$ on both machines. This setting is optimal when the repetition rate is evenly distributed across function inputs, or in the case of highly-repeating inputs that collide in the table, causing frequent evictions.

We also achieve speedups on FFT, although only for UA-ON. In this case, we achieve a speedup of $1.06 \times$ for machine $A$ across all target function variations, which are the same in this benchmark. For machine $B$, we achieve a speedup of $1.17 \times$. In contrast, we have slowdowns when updating only empty table cells. Forcing the update-always policy is beneficial in cases where the execution starts filling the empty table with input values that do not repeat as much as some other values that appear later in the execution. The FFT benchmark exhibits this behavior, which is not easy to predict unless the developer has a deep understanding of the application.

On machine $A$, every memoization version improves the performance of the original equake. We achieve modest speedups of $1.03 \times$ across all variations since they have the same functions. This benchmark has limited potential, even in the best-case scenario. As can be seen from Table 2 , the maximum speedup we could expect to achieve on machine $A$ is $1.05 \times$. Although the target functions are called frequently and exhibit high input repetition, they account for a minimal percentage of the total execution time.

On machine $B$, we achieve speedups for water_spatial with every memoization approach. As with FFT, this benchmark has better results with $U A-O N, 1.14 \times$, than with $U A-O F F, 1.08 \times$. The results are similar across target function variations, since all and math are the same, and selected ignores some functions called just once.

The barsky, ocean_cp, and typeset benchmarks present average results. We can improve performance in some cases, but it depends on the configuration and chosen target functions. The choice of targets is especially noticeable in barsky. With all, we target every function that Oddish finds, including three user-defined pure functions with low input repetition $(1.33 \%, 0.15 \%$, and $0.32 \%)$. Thus, the overhead of our approach is not offset, resulting in slowdowns. When skipping these functions, we improve the application's performance, as the remaining math functions have high input repetition.
In the case of ocean_cp, the two main target functions, pow and sin have high input repetition rates, $97.96 \%$ and $98.83 \%$, respectively, but memoizing these functions does not result in significant speedups. As illustrated by the $1.05 \times \mathrm{UB}$, there is limited speedup potential, since pow is not called often, and sin is computed quickly. Similarly, in the typeset benchmark, there is a high degree of repetition but a low amount of function calls. As such, the target functions account for a small portion of the total execution time.

We get the worst results with basicmath, fmm, sar, and susan, as we cannot improve the original application's performance with our simple memoization strategies. As shown by the UB data in Table 2, except for sar, none of these benchmarks has great potential, as the target functions account for a small portion of the total execution time. While sar appears to have great potential, none of its target functions exhibits good input repetition, they are called over 134 million times each, but their inputs repeat only 134 times.

These results represent a basic use of Oddish and the methodology. For instance, the results for the simplest strategy, Oddish (all) (UA-OFF), are achieved with the default configuration, which represents minimal effort for a developer. It is also important to highlight that achieving higher performance on some benchmarks is very dependent on configurations that are not straightforward.

\section{RELATED WORK}

Hardware memoization can target single instructions or regions such as loops or functions. Citron et al. [6] used memoization for floating-point arithmetic in media processing since data in this domain exhibits good temporal locality. The computation and table lookup are performed in parallel to mask the overhead penalty of misses. Connors et al. [7] present an approach that records the computations' results in reusable regions, which are automatically identified by their compiler, and dynamically memoized by a runtime monitor. Tsumura et al. [20] designed a memoization processor capable of targeting regions corresponding to loops and functions, with automatic detection of input and output variables.

Alvarez et al. [1] propose a mechanism based on Instruction Set Architecture (ISA) extensions to reuse the results of regions of instructions in media applications. To mitigate the memoization overhead, they rely on approximate computing and generate outputs with non-noticeable quality degradation. Arnau et al. [2] developed a hardware approximate memoization approach targeting mobile Graphics Processing Units (GPUs), exploiting the redundancy of computations in inter-frame processing. Part of the input's mantissa is removed, accelerating the hash computation and increasing the number of hits but decreasing image quality.

Since we propose a general-purpose software solution, the memoization research focused on this area is of greater importance. Hall and Mayfield [9] provide automatic software memoization for LISP applications. LISP's first-class functions and closures are used to implement a function that automatically memoizes another function. This work also allows temporarily disabling memoized versions and saving the current table in disk for later reuse. McNamee and Hall [12] developed a $\mathrm{C}++$ tool that transforms the source code of a target function, specified via a code annotation. A runtime library provides memoization support to the target function. 


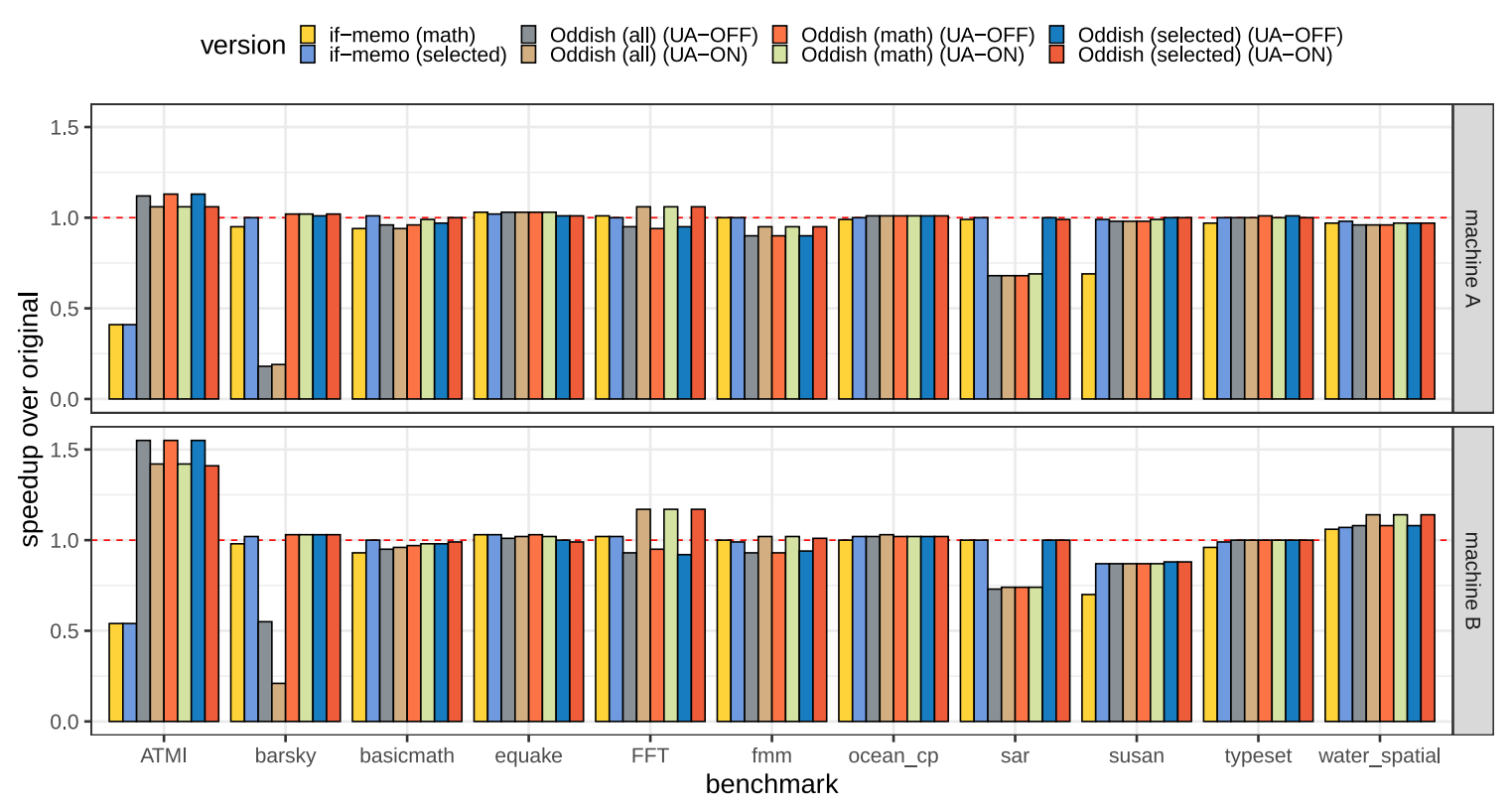

Figure 6: Speedups of the versions with memoization over the original version.

Xu et al. [21] developed a JVM implementation of memoization to test dynamic purity analysis with different purity definitions, all of which are suitable for memoization. However, not all identified purity was exploited by the memoization implementation, and they failed to achieve speedups. ATOM [16] uses Software Transactional Memory (STM) to deal with internal state and possible computation side effects, enabling memoization in object-oriented scenarios, where it is traditionally limited. The read and write logging of STM is used to track state on every method invocation. A simple method annotation is enough to mark a method as a target.

Liktor and Dachsbacher [11] propose using decoupled shading, a software rendering technique implemented in GPU local memory, to allow the memoization of the results of shading computation and improve the performance of high-quality rendering.

Suresh et al. [18] provide memoization by intercepting calls to functions that are linked dynamically, which has the advantage of not needing recompilation but is restricted on which functions it can target. Suresh et al. [19] extend the previous work scope to support developer-defined functions with an LLVM-based, compile-time approach. Like Oddish, they generate wrappers that replace the target functions and are compiled with the rest of the application, allowing for compiler link-time optimizations. This new approach is also able to identify memoizable functions automatically.

Unlike other approaches $[18,19]$, we do not limit the number of target function parameters. The chance of repetition may decrease as the number of parameters increases, but we leave this decision to the developer, as in exceptional cases, some parameters may be constants (either in the source code or in the data). Like Suresh et al. [19], we can target multiple-output functions, but we are not limited to write-only pointer parameters. However, at the moment, these functions are manually selected by the developer.

\section{CONCLUSIONS}

We presented a memoization methodology that allows developers and performance engineers to apply memoization to their applications straightforwardly. The methodology is supported by a memoization framework and several components that enable source code analyses and transformations to achieve better performance. We performed an experimental evaluation that shows performance gains on some applications, contingent on the target functions accounting for a substantial part of the application's execution time and having a high degree of input repetition. From the experiments, we can see that applications often have limited improvement potential, but we can use Oddish to evaluate this, test for corner cases, and, ultimately, apply the best configuration.

There are several different research avenues to focus on in the future. First, we would like to extend Oddish with static analyses to estimate the profitability of memoization candidates. Then, understanding how to support parallel programs can be very beneficial. Oddish supports parallelism but exclusively in read-only mode, with an offline, prefilled table. While we present an implementation and evaluation for software only, it would be interesting to have hardware-specific backends. Finally, we would like to expand the experimental evaluation with different hash functions and approximate memoization.

\section{ACKNOWLEDGMENTS}

Pedro Pinto would like to acknowledge the support provided by Fundação para a Ciência e a Tecnologia, Portugal, under Ph.D. grant SFRH/BD/141783/2018.

\section{REFERENCES}

[1] Carlos Alvarez, Jesus Corbal, and Mateo Valero. 2012. Dynamic tolerance region computing for multimedia. IEEE Trans. Comput. 61, 5 (2012), 650-665. 
[2] Jose-Maria Arnau, Joan-Manuel Parcerisa, and Polychronis Xekalakis. 2014. Eliminating Redundant Fragment Shader Executions on a Mobile GPU via Hardware Memoization. SIGARCH Comput. Archit. News 42, 3 (June 2014), 529-540.

[3] Loïc Besnard, Pedro Pinto, Imane Lasri, João Bispo, Erven Rohou, and João M.P. Cardoso. 2019. A Framework for Automatic and Parameterizable Memoization. SoftwareX 10 (2019), 100322.

[4] João Bispo and João M.P. Cardoso. 2020. Clava: C/C++ source-to-source compilation using LARA. SoftwareX 12 (2020), 100565.

[5] João M. P. Cardoso, José G. F. Coutinho, Tiago Carvalho, Pedro C. Diniz, Zlatko Petrov, Wayne Luk, and Fernando Gonçalves. 2016. Performance-driven instrumentation and mapping strategies using the LARA aspect-oriented programming approach. Software: Practice and Experience 46, 2 (2016), 251-287.

[6] Daniel Citron, Dror Feitelson, and Larry Rudolph. 1998. Accelerating multi-media processing by implementing memoing in multiplication and division units. In Proceedings of the eighth international conference on Architectural support for programming languages and operating systems. 252-261.

[7] Daniel A. Connors, Hillery C. Hunter, Ben-Chung Cheng, and Wen-Mei W. Hwu 2000. Hardware Support for Dynamic Activation of Compiler-Directed Computation Reuse. SIGPLAN Notices 35, 11 (Nov. 2000), 222-233.

[8] R. Gonzalez and R. Woods. 1992. Digital Image Processing. Addison-Wesley Publishing Company.

[9] Marty Hall and James Mayfield. 1993. Improving the performance of ai software: Payoffs and pitfalls in using automatic memoization. In Proceedings of the Sixth International Symposium on Artificial Intelligence. Citeseer, 178-184.

[10] Gregor Kiczales, John Lamping, Anurag Mendhekar, Chris Maeda, Cristina Lopes, Jean-Marc Loingtier, and John Irwin. 1997. Aspect-oriented programming. In ECOOP'97 - Object-Oriented Programming, Mehmet Akşit and Satoshi Matsuoka (Eds.). Springer Berlin Heidelberg, Berlin, Heidelberg, 220-242.

[11] Gábor Liktor and Carsten Dachsbacher. 2012. Decoupled Deferred Shading for Hardware Rasterization. In Proceedings of the ACM SIGGRAPH Symposium on Interactive $3 D$ Graphics and Games. Association for Computing Machinery, New York, NY, USA, 143-150.
[12] Paul McNamee and Marty Hall. 1998. Developing a tool for memoizing functions in C++. ACM SIGPLAN Notices 33, 8 (1998), 17-22.

[13] Donald Michie. 1968. "Memo" functions and machine learning. Nature 218, 5136 (1968), 19-22.

[14] Pedro Pinto, Tiago Carvalho, João Bispo, Miguel António Ramalho, and João MP Cardoso. 2018. Aspect Composition for Multiple Target Languages using LARA. Computer Languages, Systems \& Structures 53 (2018), 1-26.

[15] Prakash Prabhu, Stephen R. Beard, Sotiris Apostolakis, Ayal Zaks, and David I. August. 2018. MemoDyn: Exploiting Weakly Consistent Data Structures for Dynamic Parallel Memoization. In Proceedings of the 27th International Conference on Parallel Architectures and Compilation Techniques (PACT '18). Association for Computing Machinery, New York, NY, USA.

[16] Hugo Rito and João Cachopo. 2010. Memoization of methods using software transactional memory to track internal state dependencies. In Proceedings of the 8th International Conference on the Principles and Practice of Programming in Fava. 89-98.

[17] Christopher Strachey. 2000. Fundamental Concepts in Programming Languages. Higher-Order and Symbolic Computation 13, 1 (Apr 2000), 11-49.

[18] Arjun Suresh, Bharath Narasimha Swamy, Erven Rohou, and André Seznec. 2015. Intercepting Functions for Memoization: A Case Study Using Transcendental Functions. ACM Transactions on Architecture and Code Optimization 12, 2 (2015).

[19] Arjun Suresh, Erven Rohou, and André Seznec. 2017. Compile-Time Function Memoization. In 26th International Conference on Compiler Construction. Austin, TX, United States, 45-54.

[20] Tomoaki Tsumura, Ikuma Suzuki, Yasuki Ikeuchi, Hiroshi Matsuo, Hiroshi Nakashima, and Yasuhiko Nakashima. 2007. Design and evaluation of an automemoization processor.. In Parallel and Distributed Computing and Networks. $230-235$.

[21] Haiying Xu, Christopher JF Pickett, and Clark Verbrugge. 2007. Dynamic purity analysis for java programs. In Proceedings of the 7th ACM SIGPLAN-SIGSOFT workshop on Program analysis for software tools and engineering. 75-82. 